\title{
Application of Mammary Gland Endoprosthesis without a Shell in Modern Oncomammological Practice
}

\author{
S. D. Myasoyedov, D. V. Myasoyedov, P. S. Moiseyev \\ The Oncology Cathedra Clinic National Medical Academy of Postgraduate Education (NMAPE), \\ n. a. P. L. Shupyk 69 Bd., Verkhovynna Str., Kiev, Ukraine \\ Email: Superwiser@mail.ru
}

Received 2012

\begin{abstract}
Immediate and late follow-up results of oncomammological application of novel Ukrainian endoprosthesis "Nubiplant”, made of one piece of polyacrylamide gel, for mammary gland malignancies (MGM) radical treatment with reconstruction were analyzed. This endoprosthesis have proved its unique complex of favorable surgical and oncological properties, such as the shape and size accommodation for mammary gland plasty purposes, absence of shell and textured surface. Of 184 patients, operated on radically for initial stages of MGM, using "Nubiplant" endoprosthesis, postoperative complications have had occur in 18 (9,8\%): the wound seroma in $12(6,5 \%)$ and partial necrosis of cutaneous edge of postoperative wound - in $6(3,8 \%)$. The seromas were successfully treated by their punctures and local necrosis - using excision with secondary suture placement. Late follow-up results were obtained in 112 $(60,9 \%)$ patients: three-year period have had survived $104(92,6 \pm 2,4 \%)$, and a five-year one $-84(75,0 \pm 4,0 \%)$ of them. A contracture capsule was revealed in $3(2,6 \%)$ patients, and excised with the endoprosthesis change. "Nubiplant" endoprosthesis have advantages over most popular worldwide kinds of such products. It may be used securely and effectively for total or partial reconstruction of mammary gland, including cases with hypoplasia, and is more resistant to trauma, radiation therapy and local complications occurrence, including contracture capsule formation. Its property to absorb the water may be used for purposes of plastic surgery. Prophylaxis of specific immediate surgical complications is trustworthy in MGM patients, using modified endoprosthesis "Nubiplant”, containing insulin, antihistamine and antiseptic medicines.
\end{abstract}

Keywords: Mammary Gland Malignancies; Radical Surgical; Combined and Complex treatment; Endoprosthesis "Nubiplant”; Immediate and Late Results; Complications; Prophylaxis

\section{Introduction}

The optimal treatment development for mammary gland malignancies (MGM) still remain one of the main problems in medicine and biology [1, 2]. The reason for this is a steady raise of MGM incidence and mortality in majority of countries all over the world. In Ukraine, in which the same epidemiological tendency for MGM is observed, among total quantity of 960 000 cancer patients, followed today, there are 143000 , suffering MGM. Every year in Ukraine up to 15000 a newly diagnosed MGM cases are registered in women and 140-150 - in men, what constitutes 35,7 cases per 100000 of population [3]. The every year raise tendency for MGM in Ukraine coincide with a similar one in highly industrially developed countries because of a global processes impact, which are characterized by epidemiological-demographic transition situation of the population age increasing, the infectious diseases incidence lowering and chronic noninfectious diseases raising.

In Ukraine MGM holds a leading position in the oncological morbidity structure in women. Today in the country the essential tendency persists to diagnose MGM in early stages. In 2009 among newly diagnosed MGM cases $75,5 \%$ were revealed in the I-II stages and $14,3 \%$ - in the III [3]. It occurs because of nowadays greater attention in country to solving of issues, concerning prophylactic examinations conduction for mammary glands premalignancies and cancer screening, wide oncological education provided for population in mass media, paying attention to MGM early symptoms and its other clinical signs. This favorable shift in a MGM patients contingent made it possible to provide for them progressive, mainly organpreserving, strategy of treatment. Thus, organpreserving and simultaneous plastic surgical procedures have become for MGM much more, and even the most, popular [4, 5, 6, 7]. Instead of radical mastectomy universal performance, using a Patey-Dassen method, now subcutaneous mastectomy and organpreserving methods are prevailing in The Kiev's City Clinical Oncological Centre practice [7]. For these modern methods of surgical procedures various widely available mammary gland mass substituting endoprosthesis are applied, majority of which have a silicone content and a silicone shell.

After experimental investigation of endoprosthesis "Nubiplant” [8] its clinical follow-up results were analyzed [7].

\section{Materials and Methods}

Physic-chemical and clinical properties of endoprosthesis "Nubiplant" were studied in experiments on laboratory rats in accordance with the Local Ethic Committee approval [8]. Since 2005 this endoprosthesis is applied in The Clinic of the Oncology Cathedra of NMAPE n. a. P. L. Shupyk in The Kiev City Clinical Oncological Hospital. This novel Ukrainian mammary gland endoprosthesis "Nubiplant", certified in Ukraine and 
European Union, made of one piece of polyacrylamide gel and proved its apyrogenic and biocompatibility properties. This product is manufactured in various forms and sizes, and due to its physic-chemical governed changeability is promising to meet special needs of now dominating organpreserving surgery for MGM. Thus, wider application of this novel product makes possible to realize a personalized tactics and more adequate procedure choice in modern reconstructive surgical treatment of MGM. "Nubiplant” differs from analogues, because constitutes a homogenous mass without a shell and makes possible not only to compensate the whole excised mammary gland mass, but also to cut it in pieces of any form and size to substitute the mammary gland removed as a whole or any part of it after performing lumpectomy or section resection. The endoprosthesis, in accordance with experimental investigations data, as well owes complex of other specific surgical and oncological properties, such as resistance to radiation therapy adverse effects, inclination to traumatic rupture and extrusion.

In last 10 years in the Clinic there were performed 312 subcutaneous mastectomies with simultaneous mammary gland

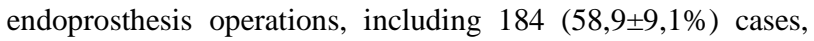
using endoprosthesis «Nubiplant», and endoprosthesis of "Mentor", "Silimed" or "McGhan" firms - in other patients. Endoprosthesis «Nubiplant» were applied predominantly for the mass restoration of a totally excised mammary gland.

In a locally spread MGM cases we prefer to perform subcutaneous mastectomy with simultaneous mammary gland endoprosthesis. Skin incision we conduct along subcutaneous plica in external direction up to anterior axillar line of a patient, what makes possible to accomplish the sentinel node intraoperative biopsy in case if it is palpable. When the sentinel node was diagnosed as free of metastatic affection, lymphadenectomy was not done. In histologically confirmed metastatic affection of regional lymph nodes the axillary lymph nodes dissection was performed in a routine fashion with excision of all three stages lymph nodes in accordance to Berg scheme. In cases of a postponed mammary gland plasty performance, using musculocutaneous flap, owing vascular pedicle, which was taken up from the back of the patient or from anterior abdominal wall, a complex restoration of the excised mammary gland mass was more precisely achieved while simultaneous application of one of these flaps and endoprosthesis "Nubiplant” of a certain size.

Thus, one of the main advantages of "Nubiplant" endoprosthesis application in reconstruction for mammary gland tumors, using organpreserving operations, comes from its uniform polyacrylamide gel content, permitting to design a smaller endoprosthesis of various size and form.

It is well known, that one of contraindications for organpreserving operations performance in surgical treatment of nodular forms of MGM is mammary gland hypoplasia. In these cases the most suitable reconstruction of the excised mammary gland portion is accomplished, using a fragment of the shell lacking kind of endoprosthesis, especially when lumpectomy is done. This procedure is performed in a following manner. The operation begins with two circular cutaneous incisions, one of which is done around areola down to the derma level and the second outside the first one by $3-4 \mathrm{~cm}$, dissecting the skin on full its width. As a next step, deepithelization of the skin around areola is conducted and separation of all mammary gland surface down to superficial fascia. A morbidly changed part of mammary gland is determined and excised completely. The equal in size and shape fragment of endoprosthesis is designed and excised from the whole "Nubiplant" endoprosthesis to substitute the mammary gland defect accurately. Using separate sutures, the edge of deepithelized part of the skin is fixed to the peeled off mammary gland cutaneous coverage by putting it above deepithelized skin. Then, the cutaneous wound edges, situated over the deepithelized part of the skin, are regularly sutured to the areola edge, using surgical thread with restricted term of absorption. As a result a uniform kind of tension between sutured cutaneous wound edges and areola is achieved, permitting to immobilize the endoprosthesis fragment preventing its disposition in late follow up period. This procedure permits to prevent also the coarse cutaneous cicatrix formation and to achieve better mass and form substituting effect, very important for the organpreserving operations outcome.

Depending on individual patient indications established, postoperative radiation therapy was prescribed in a total dose up to 42 G. In presence of metastatic affection of regional lymph nodes six courses of adjuvant chemotherapy were conducted - on the first stage, using Cooper's schedule, on the second - using docetaxel and on the third - using one preparation from a taxans group. Hormonotherapy was conducted as well - depending on the tumor receptors status, which was revealed, using histochemical investigation of biopsy material. Indication for chemotherapy conduction was also established in accordance with absence or presence of reaction (+3) of HER2-neu receptor status - while presence of such a reaction the chemotherapy course was added by herceptin preparation prescription.

Prophylaxis for postoperative morbidity in patients was achieved by perioperative prescription to them of anticoagulants (low-molecular heparins), antibiotics and analgetic preparations. In postoperative period prophylactic measures against development of lymphostasis in ipsilateral to operation site upper extremity were accomplished, using specific medicinal preparations and a local massage.

\section{The Results and Discussion}

Of 184 patients, operated on radically for initial stages of MGM, using «Nubiplant» endoprosthesis, postoperative complications have had occur in 18 (9,8\%). Of them local morbidities were presented as a local seroma in $12(6,5 \%)$ and partial necrosis of cutaneous edge of postoperative wound - in $6(3,8 \%)$. The seromas were successfully treated by their punctures and necrosis - using excision with secondary suture placement.

Contrary to majority of endoprosthesis used nowadays, "Nubiplant" do not owe the shell and the filling material inside. That's why the risk for the endoprosthesis rupture and extrusion while operational or home trauma is minimalized. "Nubiplant" endoprosthesis also have no multiple defensive layers and adhesive connection between the valve and the shell, constituting a more stable structure. They also do not have a textured surface, what lowers a rate of a contracture capsule formation.

Postoperative radiation therapy for MGM was conducted in all the patients. Of them postradiation epitheliitis have developed in 7 (3,8\%) patients, which was eliminated, using con- 
servative treatment. Before clinical application, experimental trial was conducted on laboratory animals (rats) to test radiation influence on the polyacrylamide gel state. This experimental trial have not demonstrated essential physic-chemical and structural changes in specimen of polyacrylamide gel after adjusting to them external radiation in doses of 100, 200 and 300 G. In our patients we have not observed any postradiation harmful effects on the endoprosthesis and surrounding tissues after performance of routine postoperative radiation therapy.

Also in experiment on laboratory rats was analyzed a unique property of polyacrylamide gel, depending on technological conditions applied during the process of its synthesis, to absorb, store and then continuously deliver outside endoprosthesis some chemical substances and medicines. Such properties of technologically modified variants of polyacrylamide gel, containing furacilline or antihistamine preparation, were manufactured, using the endoprosthesis 5-6 h exposition to solution, containing one of these medicines. The analysis of furacilline content in postoperative drainage exudate have shown, that all furacilline is leaving endoprosthesis completely for first 5-6 postoperative days. The endoprosthesis volume enhancement after furacilline absorption was quite mild and did not add morbidity to postoperative period course, including duration of a serous exudate leakage. We have demonstrated the best clinical effect on postoperative period course while using highly active medicines in a small volume, which were absorbed inside endoprosthesis preoperatively. It is important to apply these modified endoprosthesis individually in patients with proven risks of local surgical complications occurrence, such as inflammatory, suppuration, allergy and as a consequence of diabetes mellitus comorbidity.

For example, the selectively modified endoprosthesis was purposefully used in a young patient, suffering mammary gland adenosis, severe mastalgia and diabetes mellitus type II. In this woman a total subcutaneous mastectomy was performed on both mammary glands, 4 years between the operations, with simultaneous reconstruction, using endoprosthesis "Nubiplant", containing the absorbed insulin of prolonged activity. The immediate and late postoperative courses with follow-up were free from morbidity.

In some other patients, owing the enhanced risk of purulent-inflammatory and allergic postoperative morbidity, the endoprosthesis, containing absorbed anti-inflammatory or antihistamine medicines, accordingly, were used. These prophylactic measures have permitted to lower essentially a total rate of specific postoperative morbidity in MGM reconstructive surgery.

The next special surgical property of technologically modified "Nubiplant" endoprosthesis is its capacity to enlarge in volume step by step by 8-12 times due to water absorption from adjoining organism tissues. In experiment, the polyacrylamide gel specimen, placed in rat subcutaneously, upon several days and weeks caused the overlying skin square increase due to its significant extension above the steadily enlarging specimen volume. This property of polyacrylamide gel may be used in plastic surgery for enlargement of cutaneous flap in the donor site and as an alternative procedure for the tissue expander placement.

Endoprosthesis “Nubiplant” have uniform 0,97 specific grav- ity, thus maximally approaching that of mammary gland tissue. Such a relatively high density of "Nubiplant" endoprosthesis permits to palpate it easily and to localize, using roentgenography and CT.

The safety and security of "Nubiplant" endoprosthesis are proved by wide application for mammoplasty surgery in the Clinic, they are biocompatible and apyrogenic. Late follow-up results were obtained in $112(60,9 \%)$ patients. Three-year period have had survived $104(92,6 \pm 2,4 \%)$ patients and a five-year one - 84 (75,0 $\pm 4,0 \%)$. Remote MGM recurrences were revealed as a metastatic affection of lungs, bones and liver. These metastasis have constituted the main cause of a cancer-specific death in our patients, because palliative treatment, conducted in them, have gained mild efficacy for survival enhancement.

Among our patients a contracture capsule have developed only in $3(2,6 \%)$ of them, including twice in one patient during a ten-year follow-up period. All these patients were operated on for this complication using revision oeration - a contractile capsule excision and the endoprosthesis change with the same kind of endoprosthesis.

\section{Conclusion}

Organpreserving surgery and subcutaneous mastectomies with simultaneous mammary gland endoprosthesis operations constitute a first-line treatment option in MGM patients.

"Nubiplant" endoprosthesis have advantages over most popular worldwide kinds of such products, what was widely investigated in experimental and clinical settings. It may be used securely and effectively for total or partial reconstruction of the excised mammary gland, including cases with hypoplasia. This endoprosthesis is more resistant to trauma, radiation therapy and local complications occurrence, including contracture capsule formation. Its property to absorb the water may be used for purposes of plastic surgery. Prophylaxis of specific immediate surgical complications is trustworthy in MGM patients, using modified endoprosthesis “Nubiplant”, containing insulin, antihistamine and antiseptic medicines.

MGM radical treatment with reconstruction, using "Nubiplant”, answer adequately for a modern organpreserving mammary gland oncological surgery and combined treatment needs, guaranteeing the lowest level of specific postoperative morbidity in the aspects of prophylaxis of specific immediate and late complications.

The novel "Nubiplant” endoprosthesis application in modern oncomammological resection practice permits to restore the mammary gland mass, form and precise desired dimensions.

\section{REFERENCES}

[1] V. F. Semiglazov, S. G. Petrovskiy, and S. V. Kanayev, "Modern approaches to the treatment of mammary gland cancer," Voprosy oncologii, 2004, № 1, pp. 114-115.

[2] R. Miller,"Estrogen and breast cancer,” in Cancer of Mammary Gland, Grandess Co., Austin (USA), 1996, P. 207.

[3] Z. P. Fedorenko, A. V. Gaysenko, and L I.Gulak, "Cancer in Ukraine in 2008-2009," Bulletin of National cancer registry of Ukraine, Kyiv, 2010, № 11, P. 110

[4] N. N. Volchenko, "Morphological factors of prognosis in mam- 
mary gland cancer,” Russian oncological journal, 2000, № 3, pp. 49-52

[5] D. D. Pak and E. A. Rasskazova, "Organpreserving operations in mammary gland cancer,” 2011, № 5, pp. 10-14.

[6] A. Shi, D. Wu, X. Li, S. Zhang, S. Li, H. Xu, H. Xie, and Z. Fan, "Subcutaneous Nipple-sparing Mastectomy and Immediate Breast Reconstruction // Breast Care (Basel), 2012, Vol. 7, № 2, pp. 131-136

[7] J. D. Yang, J. W. Lee, W. W. Kim, S. O. Hwang, J. H. Jung, and H. Y. Park,"Surgical techniques for personalized oncoplastic surgery in breast cancer patients with small-to-moderate sized breasts (part 2) volume replacement // J Breast Cancer, 2012, Vol. 15, № 1, pp. 7-14

[8] S. D. Myasoyedov, Ya. A. Tersenov, and P. S. Moiseyev, “About indications for conduction of subcutaneous mastectomy with simultaneous endoprosthesis in patients, suffering mammary gland cancer,"in "The Works of XIIth Congress of oncologists of Ukraine," [Digests $12^{\text {th }}$ Congress of oncologists of Ukraine, Sudak, Crimea, p. 102, 2010].

[9] D. V. Myasoyedov, and P. S. Moiseyev, "Mammary gland endoprosthesis "Nubiplant", made of polyacrylamide gel", unpublished. 